\title{
Free non-vascularized fibular graft reconstruction after resection of metacarpal unicameral bone cyst: A case report
}

\author{
SATRIA PANDU PERSADA ISMA, ISTAN IRMANSYAH IRSAN, I GEDE MADE OKA RAHADITYA, \\ THOMAS ERWIN C.J. HUWAE and AGUNG RIYANTO BUDI SANTOSO \\ Department of Orthopaedics and Traumatology, Saiful Anwar Hospital, \\ Brawijaya University, Malang, Jawa Timur 65112, Indonesia
}

Received November 8, 2017; Accepted October 30, 2018

DOI: $10.3892 /$ mco. 2019.1803

\begin{abstract}
Unicameral bone cyst (UBC) or simple bone cyst (SBC) is a benign cystic lesion commonly found in the humerus and femur that is mainly encountered during childhood. The currently available treatments for UBC of the hand commonly involve curettage, bone grafting, partial resection with or without grafting, multiple drilling, fracture immobilization and observation alone, and steroid injection. We herein report a case treated with total resection of the cyst and non-vascularized fibular graft in a 9-year-old right-handed female patient. The patient presented with a chief complaint of a large, fast growing lump over the first metacarpal of the left hand. Flexion of the first metacarpophalangeal joint was limited. After the diagnosis was established with plain radiographs followed by biopsy, the cyst was completely removed by resecting the shaft of the metacarpal bone with preservation of the epiphyseal plate. A bone graft was obtained from the fibula and inserted in the gap, distally attached to the epiphyseal plate of the metacarpal and fixed with a Kirshner wire proximally. Radiographs revealed solid union of the bone graft to the epiphyseal plate at the head after 7 weeks, with improving function of the thumb. SBC or UBC of the metacarpal bone is very rare. A more aggressive method, such as in the case presented herein, may be necessary to treat this condition.
\end{abstract}

\section{Introduction}

Unicameral bone cyst (UBC) or simple bone cyst (SBC) is a benign cystic lesion commonly found in the humerus and femur that is mainly encountered during childhood. UBCs typically remain asymptomatic and represent $\sim 3 \%$ of all primary bone lesions (1). UBC is a serous or serosanguineous fluid-filled

Correspondence to: Dr I Gede Made Oka Rahaditya, Department of Orthopaedics and Traumatology, Saiful Anwar Hospital, Brawijaya University, Jalan Jaksa Agung Suprapto No. 2, Klojen, Malang, Jawa Timur 65112, Indonesia

E-mail: oka.rahaditya@gmail.com

Key words: unicameral bone cyst, fibular graft, metacarpal cyst cavity, which is typically inactive and remains asymptomatic until a pathological fracture occurs. The clinical presentation, location and radiographic features are rather characteristic and the diagnosis may be readily established. In $86 \%$ of the cases, UCB occurs within the first two decades of life and the proximal humerus is the most common site, whereas it is rarely observed in the hand. Very few cases of UBC have been described in the hand to date, including the metacarpals, phalanges, hamate and lunate bones. Men are twice as likely to be affected compared with women (2).

The location of UBC may be classified as active (immediately juxtaposed to the growth plate, not to be confused with Enneking stage 2 tumors) or inactive (growth plate not adjacent to the cyst) (3). On clinical examination, the lesion appears as a well-defined fluid-filled cyst in the metaphysis and diaphysis of the affected bone that may cause pain and swelling. Radiological examination is crucial for establishing the diagnosis, based on the following characteristics: Well-demarcated osteolytic lesion without marked marginal sclerosis, central location, thinning of the cortex and intralesional fracture fragment ('fallen leaf' or 'fallen fragment' sign) (3). The treatment for UBC of the hand commonly involves curettage, bone grafting, partial resection with or without grafting, multiple drilling, fracture immobilization and observation alone, and steroid injection (4).

\section{Case report}

A 9-year-old right-handed female patient presented in February 2017 to the Department of Orthopaedics and Traumatology of Saiful Anwar Hospital (Malang, Indonesia) with a chief complaint of a large, fast-growing lump over the first metacarpal of the left hand. The swelling was first observed 6 months earlier. The pain was described as dull and constant, with no diurnal variation. There was no history of trauma of infection. Physical examination revealed a firm mass, $\sim 3 \times 4 \mathrm{~cm}$ in size, which was tender on palpation, but with no local rise in temperature (Fig. 1). Flexion of the first metacarpophalangeal joint was limited. There was minimal soft tissue extension and no sensory-motor deficit; the distal circulation was also normal. On imaging, plain radiographs of the left hand revealed cystic enlargement of the first metacarpal that included the base (Fig. 2). 


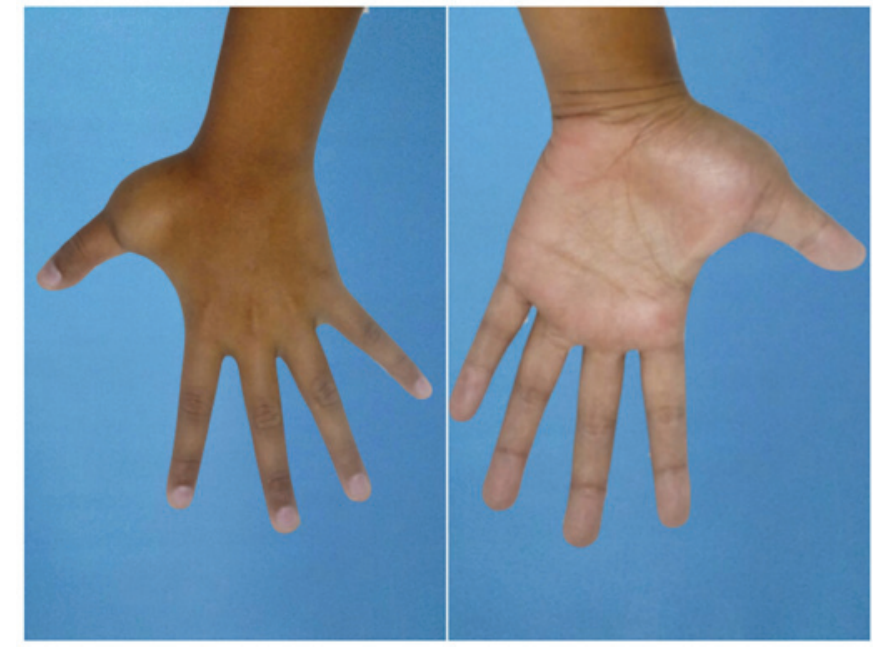

Figure 1. A 9-year-old right-handed female patient presented with a chief complaint of a large, fast-growing lump over the first metacarpal of the left hand. Physical examination revealed a firm mass $\sim 3 \times 4 \mathrm{~cm}$ in size, with limited flexion of the first metacarpophalangeal joint.

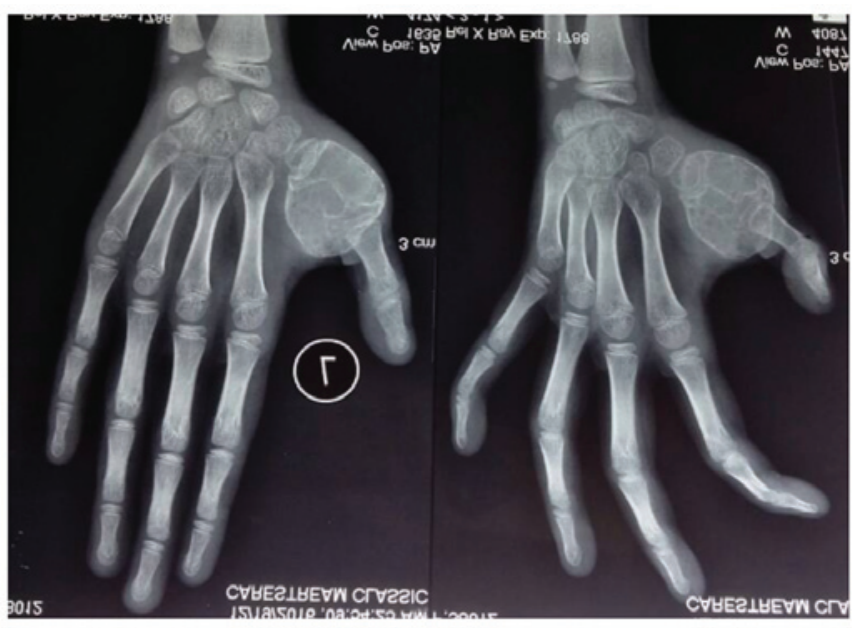

Figure 2. Anteroposterior and oblique radiograph of the left hand demonstrated a cystic enlargement of the first metacarpal bone that included its base.

A biopsy was performed, which revealed that the cyst was filled with serosanguinous fluid with a haemorrhagic tinge (Fig. 3). After the diagnosis was established, we decided to perform total resection of the cyst with placement of a non-vascularized fibular graft. The cyst was completely removed by resecting the shaft of the metacarpal with preservation of the epiphyseal plate (Fig. 4). A bone graft was obtained from the fibula and inserted in the gap, distally attached to the epiphyseal plate of the metacarpal and fixed with Kirshner wire proximally. A radiograph revealed solid union of the bone graft to the epiphyseal plate at the head after 7 weeks, with improving function of the thumb. No recurrence has been reported after the last follow-up appointment during January 2018 (Fig. 5).

\section{Discussion}

Simple bone cyst or UBC is a serous or serosanguineous fluid-filled cavity that very rarely develops in the metacarpal

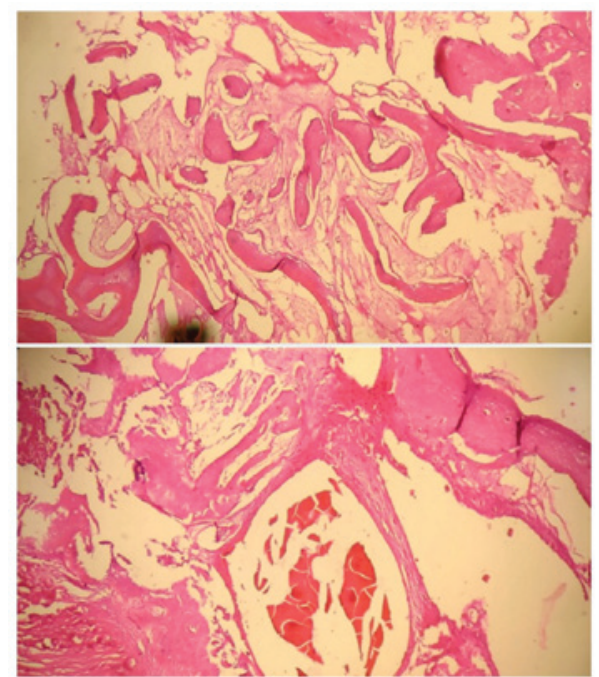

Figure 3. Photomicrograph of a biopsy specimen showing bland-appearing fibrous tissue and occasional giant cells. H\&E, hematoxylin and eosin staining; magnification, x40 and x100.

bone. The exact cause is unknown, but epiphyseal plate defect or venous outflow obstruction have been suggested as possible causes (3). Once diagnosed, UBC poses a dilemma for clinicians, as its natural history and management remain controversial. The recurrence rate of UBC ranges from 20 to $50 \%$ after various treatments (5). The differential diagnosis includes aneurysmal bone cyst and fibrous dysplasia. When additional examinations are required, magnetic resonance imaging most accurately delineates the central fluid collection. If a pathological fracture has occurred, a fluid level may be observed, mimicking the appearance of an aneurysmal bone cyst. There is no convincing evidence, however, that a UBC may transform to an aneurysmal bone cyst or other bone lesion (6). The gross pathology of UBC generally includes a singular fluid-filled cavity, unlike an aneurysmal bone cyst, which is composed of blood-filled cystic cavities that are lined by a thick, fleshy membrane with an endothelial-like inner layer. In some cases, multilocular lesions may be present. The bony architecture surrounding the cyst is non-reactive and otherwise unremarkable. Microscopic histological analysis shows a fibrous tissue membrane, with occasional giant cells present. The fluid within the cysts contains high levels of prostaglandins and enzymes (7). Traditionally, plain radiography has been used to diagnose and monitor UBCs, but advanced imaging has become more popular as technology has improved. On plain radiography, UBCs appear as lytic, expansile lesions within the medullary cavity of long bones. The cortex is thinned, but is not typically compromised structurally. Rarely, a UBC may develop in the diaphyseal portion of long bones and is known as a 'latent' cyst (8).

Among the various treatment methods mentioned above, the choice of treatment may vary according to the patient's age, the bone involved and the location of the cyst within the bone, and proper treatment should be applied to decrease the risk of recurrence $(4,5)$. Several agents have been used for intralesional injection of UBCs; Scaglietti et al first described the use of methylprednisolone injections in patients with UBCs. Recurrence rates of $15-88 \%$ have been reported after an average of three injections (9). 


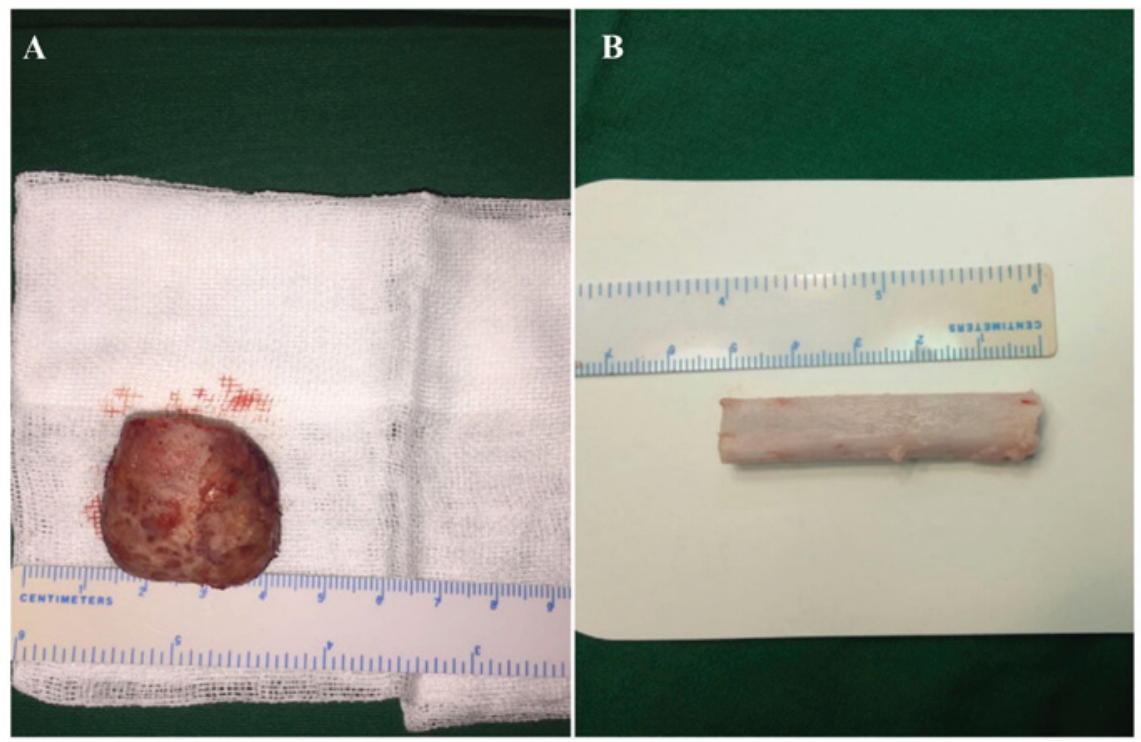

Figure 4. (A) The size of the excised cyst was $2.9 \times 3.1 \mathrm{~cm}$ (left) and the length of the graft excised from the fibula was $\sim 5.1 \mathrm{~cm}$ (right). (B) The graft was inserted in the gap, distally attached to the epiphyseal plate of the metacarpal bone, then fixed proximally with Kirshner wire.

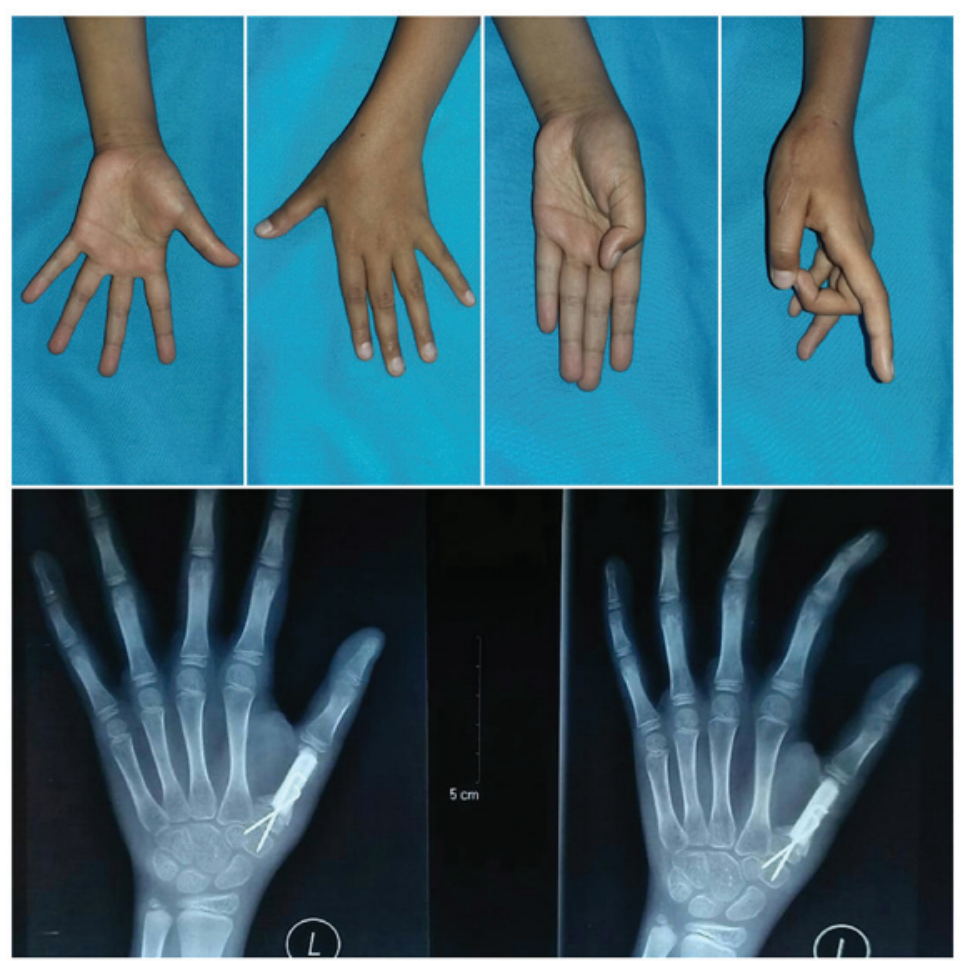

Figure 5. Postoperative X-ray and clinical picture showing the fibular graft fixed by Kirshner wire, and improved mobility of the thumb.

A more aggressive approach, such as in the present case, is occasionally necessary to treat UBC. The patient was radically treated by complete excision and a fibular graft. Our rationale was that, according to Jaffe (1), these cysts are active, as demonstrated by complete destruction of the metacarpal bone; furthermore, the patient was aged $<10$ years and was statistically more prone to recurrence (10). This method may also be used in other bone lesions, such as aneurysmal bone cyst, as reported by Gundes et al (11), in 2005.

Before proceeding with treatment, a biopsy was performed to confirm the diagnosis. We decided to perform total resection of the cyst with preservation of the periosteum and placement of a non-vascularized fibular graft. The proximal epiphyseal plate was preserved and fixed with a Kirschner wire. After 7 weeks, the function of the thumb was evaluated and union of the graft was observed. There was no recurrence after 3 months of follow-up, and the Kirschner wire was removed. The only known predictor of treatment success is the age of the patient; patients aged $>10$ years display higher rates of healing (90\%) compared with younger patients $(60 \%)$, regardless of the treatment regimen (8) due to the presence of the active growth plate in younger patients. 


\section{Acknowledgements}

Not applicable.

\section{Funding}

No funding was received.

\section{Availability of data and materials}

Not applicable.

\section{Authors' contributions}

IGMOR and TECJH were involved in the drafting of the manuscript and the revision of the manuscript for important intellectual content. ARBS and SPPI made substantial contributions to the design of the case report and were involved in the surgery. III provided final approval of the version to be published. All the authors have read and approved the final version of this manuscript.

\section{Ethics approval and consent to participate}

Ethics approval was obtained from the local Ethics Committee of Saiful Anwar Hospital.

\section{Patient consent for publication}

The legal guardians of patients included in the present study provided written, informed consent for the publication of case details and associated photographs in this manuscript.

\section{Competing interests}

The authors declare that they have no competing interests.

\section{References}

1. Jaffe HL: Tumors And Tumors Condition Of The Bone And Joints. Lea \& Febiger Publishing, Philadelphia, PA, pp629, 1958.

2. Patwardhan S, Shah K, Shyam A and Sancheti P: Simple bone cyst of metacarpal: Rare lesion with unique treatment. J Orthop Case Rep 4: 63-65, 2014.

3. Damron TA, Morris CD, Tornetta P and Einhorn TA: Oncology and Basic Science. 7th edition. Lippincott Williams \& Wikins, p140, 2008.

4. McKay DW and Nasson SS: Treatment of Unicameral bone cyst by subtotal resection without grafts. J Bone Joint Surg 55: 59-68, 1943.

5. Neer CS II, Francis KC, Johnston AD and Kiernan HA Jr: Current concepts on the treatment of solitary unicameral bone cyst. Clin Orthop Relat Res 97: 40-51, 1973.

6. Wilkins RM: Unicameral bone cysts. J Am Acad Orthop Surg 8: 217-224, 2000.

7. Komiya S, Minamitani K, Sasaguri Y,Hashimoto S, Morimatsu M and Inoue A: Simple bone cyst. Treatment by trepanation and studies on bone resorptive factors in cyst fluid with a theory of its pathogenesis. Clin Orthop Relat Res 287: 204-211, 1993.

8. Pretell-Mazzini J, Murphy RF, Kushare I and Dormans JP: Unicameral bone cysts: General characteristics and management controversies. J Am Acad Orthop Surg 22: 295-303, 2014.

9. Scaglietti O, Marchetti PG and Bartolozzi P: Final results obtained in the treatment of bone cysts with methylprednisolone acetate (depo-medrol) and a discussion of results achieved in other bone lesions. Clin Orthop Relat Res 165: 33-42, 1982.

10. Baruch A, Haas A, Lifschitz-Mercer B and Zeligowsky A: Simple bone cyst of the metacarpal. J Hand Surg Am 12: 1103-1106, 1987.

11. Gundes H, Tosun B, Muezzinoglu B and Tosun A: Total destruction of the fourth metacarpal bone by aneurysmal bone cyst: Reconstruction with strut fibular graft - a case report. Hand Surg 10: 265-269, 2005.

This work is licensed under a Creative Commons Attribution-NonCommercial-NoDerivatives 4.0 International (CC BY-NC-ND 4.0) License. 